\title{
The impact and significance of Network Speed in enhancing the usage of Unified Communications Networks Technology
}

\author{
Dr.A.Kovalan \\ Controller Of Examinations \\ Deivanaiammal College for Women \\ Villupuram Dt, Tamil Nadu
}

\author{
R.Rajini \\ Assistant Professor \&Head, \\ Department of Computer Science \\ Guru Nanak College (Autonomous), Chennai.
}

\begin{abstract}
The successfulness of digitization in business using Unified Communication Technology will lead the enterprises to next phase of business. In fact, industry experts foretell that users will have, on average, 5.5 connected UC devices by 2020. Though Unified Communication (UC) technology vendors make effort to reduce the number of complexities faced by users in finding the apt communication option and time to setup the communication, usage of UC products will face the challenge especially in traffic maintenance and in data communication speed. While the Enterprises engage UC products for communication for all purposes, bandwidth usage will become inundated. The objective of this paper is provide a marginalized solution so that in real-time the UC products deployment can be put to use effectively.
\end{abstract}

Keywords: Unified Communication, Enterprises, QOS, Bandwidth, Latency

\section{INTRODUCTION}

Unified Communication refers to all forms of communications that are exchanged via the medium of the TCP/IP network to include other forms of communications such as Internet Protocol Television and Digital Communications.

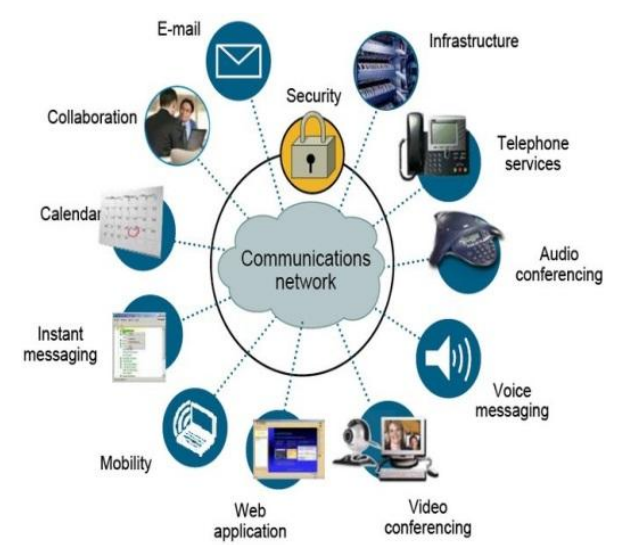

Figure I

The prime aim of any communication is breaking down the barriers. Figure I shows the Enterprises employing UC may include any combination of IP telephony, video, audio\&Web conferencing, unified messaging, instant messaging, presence, wikis, blogs, social networking and more.

It is the big task for the Enterprises to manage the network with UC products, which traditionally has handled fairly predictable and non-delay-sensitive traffic, and to have strategy to handle a combination of real-time and non-realtime applications with varying levels of performance requirements. Problems can arise with router, switch, quality of service (QoS) setting, optimization device, other applications contending for bandwidth, or a host of other issues. The router or switch related problems could be rectified by servicing or replacing them. But the key issue would be the latency in communication which adversely influences the QOS Metrics. 


\section{LITERATURE SURVEY}

The management of multifariousness and heterogeneity of the applications, services, protocols and network traffic along with their QoS requirements is a complex task to schedule their traffic through the same network paths. QoS is an vital consideration in network selection [1],[2],but it also poses a significant challenge. It refers to the capability of a network to provide better services to the users by managing the network traffic over heterogeneous technologies.

QoS parameters play important role in induction and adoption of a new Unified Communication Technology or Serviceas networks become unified and more complex [3][4].This QoS framework should classify the incoming traffic based on its requested QoS parameters, its provided QoS parameters in the access network from where it is arriving and finally the nature and requirements of the rest of traffic flows that will share the same UC session[5].

Accommodating the QoS requirements of such a complex mixture of network and applications traffic that might be sharing the same network path or even the same session is a multifaceted problem that should be tackled from different perspectives to attain optimality. Queue scheduling, session traffic mapping, traffic prioritizing are some of the technical issues that need to be carefully investigated and resolved. This challenge enforces the requirement for the dynamic scheduling mechanism to the recurrently changing needs of the demanding sessions in terms of their QoS[6].

The existing schedulers have to be modified or recreated with new constraints to fit future Unified Communication networks due to the continuous change of UC network traffic and their increasing QoS demand. To solve this, a new hybrid scheduler is proposed which employs the virtual clock concept to ensure the fairness of bandwidth allocation between different communication flows sharing the session[7].

The data flows with supreme network condition with higher channel throughput and and minimal interference will be prioritized by the opportunistic scheduler. The remedy for the this problem been suggested Proportional fair queuing (PFQ) allows to select the user with the best channel capacity status and schedules its packets for transmission. The negative aspect here is that the high capacity links will continue to utilize the link capacity and bad channelconditions links will be given only minimal opportunity to use the link capacity.The earlier schedulers are non-opportunistic and their operations are static to the changing situation of the channel or network.

The remedial scheduler for this problem is Opportunities schedulers which will allow the users to give scheduling preference with better network or channel conditions and it will increase the network overall capacity by utilizing the channel or network information available. Proportional fair queuing (PFQ) [8] is one of the popular opportunistic channel aware schedulers.

\section{ENTERPRISE UC NETWORK ARCHITECTURE}

Enterprise Unified Communication Network architecture, shown in Figure 2, includes components at different levels starting at the top from application layer, call control and conferencing layer as the middle one and Endpoint server layer at the bottom. Unity Connection, Telepresence Management Suite are the major applications are to be developed at this layer and the services and tools required for call control as Instant Message \& Presence and UC Manager are to be used and Telepresence Server is 
suppose to be setup exclusively for handling this service at conference layer. All the components are to be connected toUC Manager tool which will monitor and manage all the services connected within and outside Enterprise using Internet and PSTN/ISDN.

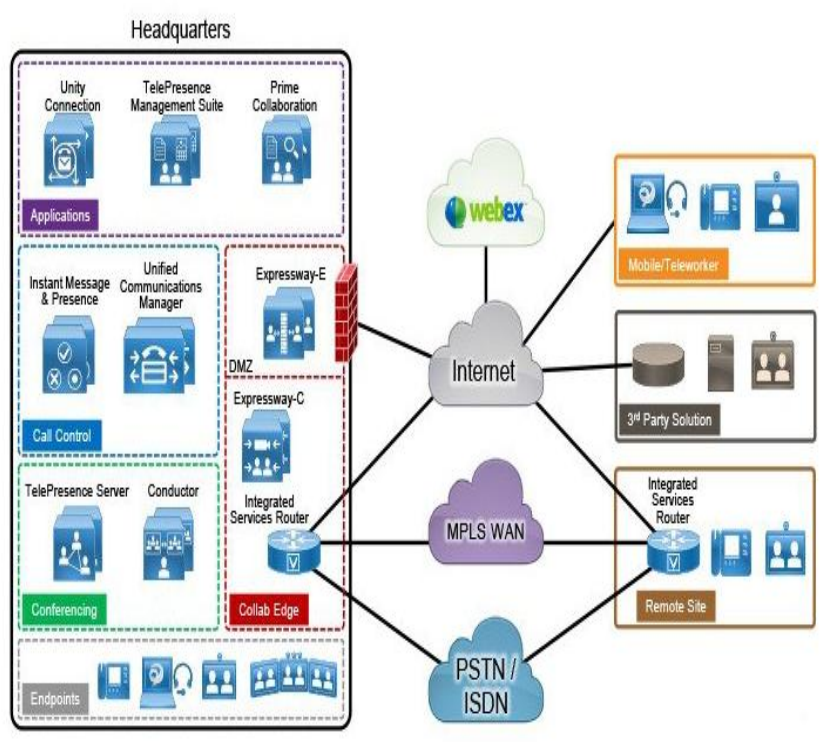

\section{NETWORK TRAFFIC} MONITOR SYSTEM(NTMS)

An Enterprise UC infrastructure requires resilient, agile, secure and simple features which should be modified as per be need dynamically. The Unified services are enabled using the key components such as Registrar and Call Routing Server, Sessiion Border Controller, Media Gateway, Video Conference Bridges, Collaboration application tools and Endpoints etc., Though several challenges are to be faced in deploying UC, an important issue which is to be considered is network speed which will lead to latency. It is the one which will directly impact the entire communication network of the Enterprise. To regulate and improvise the flow of communication, a methodology called The Enterprise has to maintain a tool called Network Traffic
Monitor System(NTMS) which keeps track of all incoming and outgoing data through network. Also It maintains a detailed report of source and destination IP addresses from and to the data transferred, volume of data, time duration of data transfer etc., These details will be applied in the algorithm for finding out the frequency level of data transmission of a particular IP address. The report generated by the algorithm will be used by the network administrator of the respective Enterprise to make decision in giving permission for IP addresses to transfer the data[7]. The following is the algorithm for Load Balancing.

\section{Load Balancing Algorithm :}

Step 1: Capture the incoming and Outgoing Data flow with IP addresses using NMTS for specified duration(Especially peak hours).

Step 2: Store the Report in MS-Access table.

Step 3: Execute a for-loop, and generate a report with list of users . Fix the priority range for them based on data they are using and as per enterprise norms.

Step 4: Based on the Priority List, the lower level prioritized users have to be stopped from giving permission to use the network.

Step 5: Now the Network traffic will be reduced and most prioritized users can have required Network speed to transmit the data.

\section{Conclusion}

In present scenario, medium level enterprises engage Unified Communication Products for their day to day business activities and transactions as it highly reduces travelling expenses and time factor. But the hindrance they face is network speed at peak time as they have to wait for longer time to get their data transmitted. In case of video, audio 
conference, they tend to suffer with poor quality of video/audio. This problem can be reduced to maximum possible by using Load Balancing Technique in their enterprises. Instead of going for upgradation say for example from $2 \mathrm{G}$ to $3 \mathrm{G}$, now from $3 \mathrm{G}$ to $4 \mathrm{G}$ network speed, Enterprise can balance the network traffic which improves the bandwidth so that the prioritized users can avail that bandwidth which will enhance the network speed.

\section{REFERENCE}

[1]Tanenbaum AS, "Computer Networks", Prentice Hall Publications, 4th ed., ISBN0130661023

.[2] Forouzan BA, "TCP/IP: Protocol Suite", Tata McGraw - Hill Publishing Company Limited, 1 st ed.

[3]National Research Council, "Networking Health:Prescriptions for the Internet", The National Academies Press: Washington, DC (2000)

[4] Research on Application of perceived QoS Guarantee through Infrastructure Specific Traffic Parameter Optimization.ISSN: 2074-9090 (Print), ISSN: 2074-9104 (Online) IJCNIS Vol.6, No.3, Feb. 2014

[5]Real-time traffic classification for unified communication networksConference Paper - August 2013 DOI:10.1109/MoWNet.2013.6613788

[6] Simulation Based Comparative Performance Analysis of QoS Traffic Scheduling using Fair and Delay Adaptive Scheduler (FDAS) versus WFQ and EDF 2016 13th IEEE Annual Consumer Communications \& Networking Conference (CCNC)

DOI:10.1109/CCNC.2016.7444910
[7] Fengxiang Zhang and A Burns, "Schedulability Analysis for Real-Time Systems with EDF Scheduling," IEEE Transactions on Computers, vol. 58, no. 9, pp. 1250-1258, Spt 2009.

[8] Matthew Andrews and K. Ramanan, A. Stoyar and Phil Whitting K. Kumaran, "Providing Quality of Service over a Shared Wireless Link," IEEE Communications, vol. 39, no. 2, pp. 150154, Feb 2001. 